\title{
The nanocomposite fullerol reduces oxidative stress, pulmonary injury, and mortality in a rat model of acute lung injury
}

\author{
Rosária Aires $^{1}$, Ildernandes Vieira-Alves ${ }^{1}$, Leda Maria Coimbra-Campos ${ }^{1}$, Marina Ladeira ${ }^{1}$, \\ Teresa Socarras $^{1}$, Paula Campos ${ }^{1}$, Silvia Guatimosim ${ }^{1}$, Luiz Ladeira ${ }^{1}$, Steyner Cortes ${ }^{1}$, and \\ Virginia Lemos $^{1}$ \\ ${ }^{1} \mathrm{UFMG}$
}

September 24, 2021

\begin{abstract}
BACKGROUND AND PURPOSE Acute lung injury (ALI) is a critical disorder that has high mortality rates, and pharmacological therapies are so far ineffective. The pathophysiology of ALI involves pulmonary oxidative stress and inflammatory response. Fullerol is a carbon nanocomposite that possesses antioxidant and anti-inflammatory properties. Here, we evaluated the therapeutic potential of fullerol and its mechanisms in a model of paraquat-induced ALI. EXPERIMENTAL APPROACH Rats were divided into ALI (paraquat alone), fullerol (paraquat plus fullerol), and control groups. Survival curves were estimated using the Kaplan-Meier method. The myeloperoxidase assay, ELISA, and hematoxylin and eosin staining were used to determine neutrophils infiltration, cytokines production, and histopathological parameters in lung samples, respectively. The antioxidant effect of fullerol was evaluated in vitro and ex vivo. KEY RESULTS Fullerol (0.01 to $0.3 \mathrm{mg} / \mathrm{kg})$ markedly reduced the severe lung injury and high mortality rates observed in ALI rats. Moreover, fullerol $(0.03 \mathrm{mg} / \mathrm{kg})$ inhibited the reactive oxygen species formation and lipid peroxidation seen in lungs from ALI rats, and exhibited a potent concentration-dependent $\left(10^{-4}\right.$ to $\left.10^{-1} \mathrm{mg} / \mathrm{ml}\right)$ in vitro antioxidant activity. Importantly, fullerol $(0.03 \mathrm{mg} / \mathrm{kg})$ inhibited neutrophils accumulation in bronchoalveolar lavage and lungs, and the increase in pulmonary levels of TNF- $\alpha$, IL-1 $\beta$, IL- 6 , and CINC-1 in ALI rats. CONCLUSIONS AND IMPLICATIONS Fullerol treatment was effective in reducing pulmonary damage and ALI-induced mortality, highlighting its therapeutic potential in an ALI condition. Searching for new pharmacological therapies to treat ALI may be desirable especially in view of the new coronavirus disease 2019 that currently plagues the world.
\end{abstract}

\section{Hosted file}

Manuscript_BJP.pdf available at https://authorea.com/users/435742/articles/538554-thenanocomposite-fullerol-reduces-oxidative-stress-pulmonary-injury-and-mortality-in-a-ratmodel-of-acute-lung-injury 